TRANSACTIONS OF THE

AMERICAN MATHEMATICAL SOCIETY

Volume 281. Number 2. February 1984

\title{
A PROPERTY OF COMPLETE MINIMAL SURFACES \\ BY
}

\author{
THOMAS HASANIS AND DIMITRI KOUTROUFIOTIS
}

\begin{abstract}
If $M$ is a complete minimal surface in $R^{n}$, we denote by $W$ the set of points in $R^{n}$ that do not lie on any tangent plane of $M$. By taking a point in $W$ as origin, the position vector of $M$ determines a global unit normal vector field $e$ to $M$. We prove that if $e$ is a minimal section, then $M$ is a plane. In particular, the set of tangent planes of a nonflat complete minimal surface in $R^{3}$ covers all $R^{3}$. We also prove a similar result for a complete minimal surface $M$ in $S^{3}$, and deduce from it that if the spherical image of $M$ lies in a closed hemisphere, then $M$ is a great $S^{2}$.
\end{abstract}

Introduction. Consider a connected and complete surface $M^{2}$ in euclidean space $R^{3}$. The union of its tangent planes covers a certain part of the ambient space. We denote by

$$
W=R^{3}-\bigcup_{p} T_{p} M^{2}
$$

the set of points that are omitted. For what $M^{2}$ is $W$ empty?

To begin with, we observe that $W$ is empty if $M^{2}$ is nonorientable. For if $W$ contains a point $o$, the position vector of $M^{2}$ with origin $o$ is different from zero and transversal to $M^{2}$. By taking at its endpoint the component perpendicular to $M^{2}$, we obtain a global nonzero normal vectorfield, so $M^{2}$ is orientable.

For compact $M^{2}$ the question is essentially one of differential topology and has a complete answer. A theorem of Halpern [4] states that, for a compact $n$-dimensional manifold $M^{n}$ immersed in $R^{n+1}$, the set $W$ is not empty if and only if $M^{n}$ is imbedded as the boundary of an open star-shaped set. In particular, $W$ is empty for all compact surfaces of genus greater than 0 in $R^{3}$, and all those of genus zero that have self-intersections.

If $M^{2}$ is not assumed to be compact, a general answer to the question does not seem easy. Here we remark that, whereas in the compact case the set $W$ is obviously open, this need not be the case for a noncompact $M^{2}$. It is easy to construct examples of imbedded noncompact complete surfaces for which $W$ is not empty, and neither open nor closed. These surfaces are the boundaries of appropriate unbounded star-shaped sets; they can be chosen so that their curvature throughout is negative, nonpositive, or of variable sign. If $M^{2}$ has nonnegative curvature, then $W$

Received by the editors December 3, 1982 and, in revised form, March 3, 1983. The contents of this paper have been presented in a lecture delivered by the second author at the Brazilian IV. School of Differential Geometry, held at IMPA, Rio de Janeiro, in July 1982.

1980 Mathematics Subject Classification. Primary 53C42; Secondary 53A10.

Key words and phrases. Minimal surface, tangent plane, minimal section, support function.

(C)1984 American Mathematical Society $0002-9947 / 84 \$ 1.00+\$ .25$ per page 
is, of course, the whole convex interior of $M^{2}$. There are also examples of complete surfaces with self-intersections whose tangent planes do not cover all $R^{3}$ : cylinders, say, over plane curves with self-intersections. The one-sheeted hyperboloid of revolution is an example where $W$ consists of one point.

The foregoing considerations suggest that the class of complete surfaces with nonempty $W$ is very large. Nevertheless, we shall show in the next section that it does not include any nonflat minimal surfaces. In other words, a complete minimal surface whose set of tangent planes omits a point in $R^{3}$ must be the plane. The proof of this result is inspired by that of Theorem 1 in [5], the crucial point being a recent result of Fischer-Colbrie and Schoen concerning positive solutions of certain elliptic equations on the hyperbolic disc [2, p. 205, Corollary 3]. So the proof strongly uses the assumption that $M^{2}$ is two dimensional. It would be interesting to know what conclusions can be drawn from $W \neq \varnothing$ in the case of a higher-dimensional minimal hyeprsurface.

In the last section of this article, we consider the same problem for a minimal surface $M^{2}$ in the 3-sphere $S^{3}$. Here an analogous result holds, which can also be formulated as a statement concerning the spherical image of $M^{2}$. With a little additional effort, we are able to prove that a complete surface, immersed minimally in $S^{3}$, must be a great $S^{2}$ in case its spherical image lies in a closed hemisphere of $S^{3}$.

We shall assume connectivity of all manifolds and smoothness of all mappings under consideration.

Immersion in euclidean space. The geometrical question posed in the introduction can be formulated as a question concerning existence of positive solutions for a certain differential equation; the reason for this is a simple observation: $W \neq \varnothing$ is equivalent to the existence of a point with respect to which the support function of $M^{2}$, considered as a function on $M^{2}$, is positive. We shall begin, therefore, by deriving certain formulae that involve the support function.

Our result about minimal surfaces in $R^{3}$ will be a corollary of a more general theorem concerning certain immersions of arbitrary codimension. So we start within a general framework. Let $M^{n}$ be an $n$-dimensional Riemannian manifold, and $\varphi$ : $M^{n} \rightarrow R^{k+n}$ an isometric immersion. We make the usual identification of the tangent space of $M^{n}$ with its image under $d \varphi$; this image is considered as an $n$-dimensional linear variety of $R^{k+n}$. Denote by $W$ the set of points in $R^{k+n}$ that do not lie on any tangent space of $M^{n}$. We assume $W$ is not empty and pick a point $o$ from it as origin. Now we decompose the position vector $x$ of a point $p \in M^{n}$ in a component $x_{T}$ tangent to $\varphi\left(M^{n}\right)$, and a component $x_{N}$ normal to $\varphi\left(M^{n}\right)$ :

$$
x=x_{T}+x_{N} .
$$

This decomposition takes place in the tangent space of $R^{k+n}$ at the point $\varphi(p)$, where $x$ is identified canonically with a vector in that tangent space. By assumption we have $x_{N} \neq 0$; so we may set $x_{N}=f e$, where $f=\left|x_{N}\right|$ is a smooth positive function on $M^{n}$, and $e$ is a global unit normal vectorfield on $M^{n}$. We follow [1, p. 60] in calling $f$ the canonical support function of $M^{n}$, and $e$ the canonical normal vectorfield of $M^{n}$ corresponding to the origin $o$. 
Let $\bar{\nabla}$ be the standard connection of $R^{k+n}, \nabla$ the Riemannian connection of $M^{n}$, and $\alpha$ the second fundamental form of the immersion. For tangent vectors $X$ and $Y$ of $M^{n}$, we have the Gauss formula

$$
\bar{\nabla}_{X} Y=\nabla_{X} Y+\alpha(X, Y)
$$

and the Weingarten formula

$$
\bar{\nabla}_{X} e=-A X+\nabla_{X}^{\perp} e
$$

where the $(1,1)$-tensor $A$ (Weingarten mapping) associated with $e$ is given by $\langle A X, Y\rangle=\langle\alpha(X, Y), e\rangle$, and $\nabla^{\perp}$ is the connection in the normal bundle. Using these we compute

$$
\begin{aligned}
X & =\bar{\nabla}_{X} x=\bar{\nabla}_{X} x_{T}+(X f) e+f \bar{\nabla}_{X} e \\
& =\nabla_{X} x_{T}+\alpha\left(X, x_{T}\right)+(X f) e+f\left(-A X+\nabla_{X}^{\perp} e\right) .
\end{aligned}
$$

Taking the tangential component of both sides of this equation, we obtain

$$
\nabla_{X} x_{T}=(I+f A) X
$$

where $I$ is the identity. Therefore, the tangent vectorfield $x_{T}$ of $M^{n}$ has divergence

$$
\operatorname{div} x_{T}=n+f \operatorname{trace} A \text {. }
$$

Taking the normal component of (1.1), we obtain

$$
\alpha\left(X, x_{T}\right)+\langle X, \operatorname{grad} f\rangle e+f \nabla_{X}^{\perp} e=0 .
$$

Let $\left\{e_{1}, e_{2}, \ldots, e_{k}\right\}$ be a local orthonormal frame field in the normal bundle of $M^{n}$, with $e_{1}=e$. Scalar multiplication of (1.4) with $e$ gives

$$
\operatorname{grad} f=-A x_{T} \text {. }
$$

The normal component in the Weingarten equation can be written as

$$
\nabla_{X}^{\perp} e=\sum_{\beta=2}^{k} s_{1 \beta}(X) e_{\beta}, \quad s_{1 \beta}(X)=\left\langle\nabla_{X}^{\perp} e, e_{\beta}\right\rangle .
$$

Let $A_{\beta}$ denote the $(1,1)$-tensorfield associated with $e_{\beta}$. Taking the component of (1.4) in the direction of $e_{\beta}$, we obtain for every $X$,

$$
\left\langle A_{\beta} x_{T}, X\right\rangle+f \cdot s_{1 \beta}(X)=0, \quad \beta=2, \ldots, k .
$$

We shall now compute the Laplacian of $f$. Using the decomposition of $x$ and $f=\langle x, e\rangle$, we have $X f=-\langle x, A X\rangle$, and so $\left(\nabla_{X} X\right) f=-\left\langle x, A\left(\nabla_{X} X\right)\right\rangle$. Using $\left\langle x, e_{\beta}\right\rangle=0$ for $\beta \geqslant 2$, we have

$$
X(X f)=-\langle X, A X\rangle-\left\langle x, \nabla_{X}(A X)\right\rangle-\langle A X, A X\rangle f .
$$

Therefore,

$$
\begin{aligned}
\nabla^{2} f(X, X) & =X(X f)-\left(\nabla_{X} X\right) f \\
& =-\langle X, A X\rangle-\langle A X, A X\rangle f-\left\langle x,\left(\nabla_{X} A\right) X\right\rangle .
\end{aligned}
$$


Taking the trace of $\nabla^{2} f$, we obtain

$$
\Delta f+\operatorname{trace}\left(A^{2}\right) f+\operatorname{trace} A=-\left\langle x, \sum_{i=1}^{n}\left(\nabla_{X_{i}} A\right) X_{i}\right\rangle,
$$

where $\left\{X_{1}, \ldots, X_{n}\right\}$ is a local orthonormal frame field of $M^{n}$.

In order to bring the right-hand side of this last equation to an amenable form, we use the Codazzi equation

$$
\left(\nabla_{X} A\right) Y-\left(\nabla_{Y} A\right) X=\sum_{\beta=2}^{k}\left[s_{1 \beta}(X) A_{\beta} Y-s_{1 \beta}(Y) A_{\beta} X\right] .
$$

Since $A$ is selfadjoint, so is $\nabla_{X} A$; furthermore $X(\operatorname{trace} A)=\operatorname{trace}\left(\nabla_{X} A\right)$, as is well known. Using all this we compute for an arbitrary tangent vector field $Y$ of $M^{n}$,

$$
\begin{aligned}
\sum_{i}\left\langle\left(\nabla_{X_{i}} A\right) X_{i}, Y\right\rangle= & \langle\operatorname{grad}(\operatorname{trace} A), Y\rangle-\sum_{\beta} s_{1 \beta}(Y) \operatorname{trace} A_{\beta} \\
& +\sum_{i} \sum_{\beta} s_{1 \beta}\left(X_{i}\right)\left\langle A_{\beta} X_{i}, Y\right\rangle
\end{aligned}
$$

If $M^{n}$ has codimension 1 the two summands on the right of (1.9) are absent, so that (1.8) becomes

$$
\Delta f+\operatorname{trace}\left(A^{2}\right) f+\operatorname{trace} A=-\langle x, \operatorname{grad}(\operatorname{trace} A)\rangle .
$$

REMARK 1. Formula (1.10) is, in fact, valid under the following assumptions: $M^{n}$ is orientable in $R^{n+1}, e$ is a unit normal vectorfield on $M^{n}$, and $f=\langle x, e\rangle$ with respect to some chosen origin. There is no need to assume $o \in W$; the set $W$ may be empty. This is so because we still have the decomposition $x=x_{T}+f e$, and the proof of all the previous numbered equations goes through.

We continue with the case of arbitrary codimension. We have by (1.7), since $f$ is never zero,

$$
s_{1 \beta}(Y)=-(1 / f)\left\langle A_{\beta} x_{T}, Y\right\rangle, \quad \beta \geqslant 2 .
$$

Replacing $s_{1 \beta}$ by this expression in (1.9), we deduce

$$
\begin{aligned}
\sum_{i}\left(\nabla_{X_{i}} A\right) X_{i}= & \operatorname{grad}(\operatorname{trace} A)+\frac{1}{f} \sum_{\beta \geqslant 2}\left(\operatorname{trace} A_{\beta}\right) A_{\beta} x_{T} \\
& -\frac{1}{f} \sum_{i} \sum_{\beta \geqslant 2}\left\langle A_{\beta} x_{T}, X_{i}\right\rangle A_{\beta} X_{i} ;
\end{aligned}
$$

therefore,

$$
\begin{aligned}
\left\langle x, \sum_{i}\left(\nabla_{X_{i}} A\right) X_{i}\right\rangle= & \langle x, \operatorname{grad}(\operatorname{trace} A)\rangle+\frac{1}{f} \sum_{\beta \geqslant 2}\left(\operatorname{trace} A_{\beta}\right)\left\langle A_{\beta} x_{T}, x_{T}\right\rangle \\
& -\frac{1}{f} \sum_{\beta \geqslant 2}\left\langle A_{\beta} x_{T}, A_{\beta} x_{T}\right\rangle .
\end{aligned}
$$


Rearranging terms we finally obtain

$$
\begin{aligned}
& \Delta f+\operatorname{trace}\left(A^{2}\right) f+\operatorname{trace} A \\
& \quad=-\langle x, \operatorname{grad}(\operatorname{trace} A)\rangle+\frac{1}{f} \sum_{\beta=2}^{k}\left\langle\left(A_{\beta}-\left(\operatorname{trace} A_{\beta}\right) I\right) x_{T}, A_{\beta} x_{T}\right\rangle .
\end{aligned}
$$

We shall use the terminology introduced in [10]; a normal vectorfield $e$ of an immersion of $M^{n}$ in a space form is called a minimal section if it is parallel in the normal bundle of $M^{n}$, that is $\nabla_{X}^{\perp} e=0$ for every tangential $X$, and the Weingarten mapping $A$ defined by $e$ satisfies trace $A=0$.

THEOREM 1. Let $M^{2}$ be a complete two-dimensional manifold, and $\varphi: M^{2} \rightarrow R^{k+2}$ an isometric immersion. Assume $W$ is not empty and pick a point in $W$ as origin. If the corresponding canonical normal vectorfield is a minimal section, then $\varphi$ is an imbedding and $\varphi\left(M^{2}\right)=R^{2}$.

PROOF. In the notation introduced above, we have

$$
x=x_{T}+f e, \quad f=\langle x, e\rangle>0 .
$$

Because $e$ is parallel in the normal bundle, we have $s_{1 \beta}=0$; therefore, (1.7) implies $A_{\beta} x_{T}=0$ for $2 \leqslant \beta \leqslant k$. Now (1.11) reduces to

$$
\Delta f+\operatorname{trace}\left(A^{2}\right) f=0 .
$$

Consider the tangential vectorfield $x_{T}$ on $M^{2}$; by (1.3), the set of points $C$ where $x_{T}=0$ has empty interior. We write the Gauss equation in the form

$$
K-\operatorname{det} A=\sum_{\beta=2}^{k} \operatorname{det} A_{\beta},
$$

where $K$ is the Gaussian curvature of $M^{2}$. Since det $A_{\beta}=0$ locally on the complement of $C$, and the right-hand side of this equation is well defined on all $M^{2}$, by continuity it vanishes identically on $M^{2}$. So the Gauss equation takes the form $K=\operatorname{det} A$ and, using trace $A=0$, we rewrite it as

$$
\operatorname{trace}\left(A^{2}\right)=-2 K \text {. }
$$

Clearly, it is sufficient to prove the theorem for the universal Riemannian covering surface of $M^{2}$. So we assume, in addition, that $M^{2}$ is simply connected. By means of isothermal coordinates, we may now view $M^{2}$ as a Riemann surface. According to Koebe's uniformization theorem, there are three possibilities for $M^{2}$ :

(a) $M^{2}$ is compact. This is impossible by (1.3) and Stokes' theorem.

(b) $M^{2}$ is conformally equivalent to the unit disc in the complex plane (hyperbolic). In that case the equation

$$
\Delta f-2 K f=0
$$

on the disc would have a positive solution; that is impossible by Corollary 3 of [2] which states that the equation $\Delta f-a K f+P f=0$ has no positive solution on the hyperbolic disk in case $a \geqslant 1$ is a constant and $P$ a nonnegative function. 
(c) $M^{2}$ is conformally equivalent to the complex plane (parabolic). Because $K \leqslant 0$ by (1.12), we infer from (1.13) that $\Delta f \leqslant 0$. Thus, $f$ is a positive superharmonic function on the complex plane, so $f=$ const $>0$. From (1.13) we now have $K=0$, hence $A=0$ by (1.12).

Consider the vectorfield $x_{T}$ in the neighbourhood of a point where $x_{T} \neq 0$; we maintain that its integral curves are, when suitably parametrized, geodesics of $M^{2}$. We set $x_{T}=g v,|v|=1$. Then, on the one hand, $\nabla_{v} v \perp v$, while on the other, $\nabla_{v} v$ is collinear with $v$ by virtue of (1.2) with $X=v$; so $\nabla_{v} v=0$. Furthermore, the second fundamental form $\alpha$ of $M^{2}$ vanishes on $v$ because $A=0$ and $A_{\beta} x_{T}=0$, so we conclude from the Gauss formula that $\bar{\nabla}_{v} v=0$; this means the integral curves of $x_{T}$ have images that are straight lines in $R^{k+2}$.

From the Weingarten equation we deduce $\bar{\nabla}_{X} e=0$ for every tangent vector $X$ of $M^{2}$; thus, the normal vectorfield $e$ is constant along $\varphi\left(M^{2}\right)$.

We now consider the vector $f e$ with the origin as initial point and take its endpoint $q_{0}$ as the new origin. The new position vector of a point $\varphi(p)$ is equal to $x(p)-f e=x_{T}(p)$. Pick a point $p \in M^{2}$ such that $\varphi(p)=q \neq q_{0}$; the position vector of $q$ is now the tangent vector $x_{T}(p) \neq 0$. Let $\gamma$ denote the complete geodesic of $M^{2}$ through $p$ in direction $x_{T}(p)$. We know already that a part of $\gamma$ near $p$ is mapped isometrically into the straight line $l$ determined by the new position vector of $q$. If, starting from $p$, a point moves with unit speed along $\gamma$ in direction $-x_{T}(p)$, then the image point moves with unit speed along $l$ from $q$ towards $q_{0}$. Along $\gamma$ we will certainly encounter a point $p_{0}$ with $x_{T}\left(p_{0}\right)=0$; for, otherwise, the half-line from $q$ towards $q_{0}$ would be in the image of $\gamma$, but not the point $q_{0}$, which is absurd. So $q_{0}=\varphi\left(p_{0}\right)$ and the position vector from $q_{0}$ to $q$ lies in $\varphi\left(M^{2}\right)$. It follows immediately that $\varphi\left(M^{2}\right)$ lies entirely in an $R^{2}$ which is the tangent plane to $\varphi\left(M^{2}\right)$ at $q_{0}$. So $\varphi$ is a local isometry of $M^{2}$ into $R^{2}$. Because $M^{2}$ is complete, $\varphi$ must be surjective; and because any two points of $R^{2}$ can be joined by a unique geodesic, $\varphi$ must be injective. This completes the proof of Theorem 1 .

The result we just proved indicates that the extrinsic geometry of an immersion of high codimension is strongly influenced by the behaviour of the canonical normal vectorfield $e$. Theorem 1 is false if, instead of $e$, we take another normal vectorfield, all other assumptions remaining. To see this, consider a nontrivial complete oriented minimal surface in $R^{3}$. We view $R^{3}$ as a certain subspace of $R^{4}$; then $W$ is not empty and the normal vectorfield of the surface in $R^{3}$ is a minimal section in $R^{4}$.

REMARK 2. The union of all linear varieties tangent to $\varphi\left(M^{n}\right)$ is the image of the tangent bundle $T M^{n}$ under the mapping $F: T M^{n} \rightarrow R^{n+k}, F(p, v)=\varphi(p)+d \varphi_{p}(v)$. According to Sard's lemma, this union has measure zero if $n<k$. Thus, $W$ is dense in $R^{n+k}$ for $n<k$. In the particular case of surfaces, the set $W$ may perhaps be empty only for immersions in $R^{3}$ or in $R^{4}$.

In order to furnish some justification for our assumptions in Theorem 1, we now give an example due to L. Rodriguez. Let $f(z)$ be an entire holomorphic function; it defines a complete minimal surface in $R^{4}$ as the complex graph $\varphi: C \rightarrow C \times C=R^{4}$, $\varphi(z)=(z, f(z))$. We see immediately that the point $(0,0) \in R^{4}$, say, lies on some tangent plane of the surface if and only if there exists a $z$ satisfying the equation 
$f(z)-z f^{\prime}(z)=0$. If, therefore, the entire function $f(z)-z f^{\prime}(z)$ omits the value 0 , then $W$ is not empty. Take the entire function $e^{z^{2}}:$ it is never zero. We consider the differential equation

$$
f(z)-z f^{\prime}(z)=e^{z^{2}}
$$

and try to find an entire solution. Expanding both sides in power series around the origin and comparing coefficients we easily establish that

$$
f(z)=1-\sum_{n=1}^{\infty} \frac{z^{2 n}}{n !(2 n-1)}
$$

is a solution. Clearly, this series converges for all $z$, so $f(z)$ is an entire function. Thus, we have an example of a complete nonflat minimal surface in $R^{4}$, whose tangent planes do not cover all $R^{4}$. The normal bundle of this surface has curvature tensor $R^{\perp}$ that can be zero at isolated points only. In order to explain this statement, we recall some known facts. Quite generally for surfaces in $R^{4}$, the zeros of $R^{\perp}$ are the points where the normal curvature vanishes or, equivalently, where the ellipse of curvature degenerates; consult [6] on this. Now a straightforward computation shows the ellipse of curvature of the graph in $R^{4}$ of a holomorphic function $f(z)$ is always a circle (Eisenhart 1912, see [6, p. 275]) and its radius is zero exactly at the points $z$ where $f^{\prime \prime}(z)=0$. So $R^{\perp}$ can be zero at isolated points at most if $f$ is not linear. From this it follows, because the surface has codimension 2 , that no unit normal vectorfield to a complex-analytic graph in $R^{4}$ can be parallel in the normal bundle, not even locally, unless the surface is a plane. In particular, the minimal surface we have just constructed possesses no unit normal vectorfield that is parallel in the normal bundle. The reader should bear this example in mind in assessing the necessity of the assumption on the normal bundle in Theorem 1; it would be interesting to know if it can be weakened, at least in the case of minimal surfaces.

In the case of immersions of codimension 1, there is essentially only one unit normal vectorfield, and it is automatically parallel in the normal bundle; so we obtain

COROllary 1. A complete minimal surface in $R^{3}$ which is not a plane has this property: the union of its tangent planes covers all $R^{3}$.

REMARK 3. We point out that the proof of Theorem 1 becomes much simpler in the most important special case of Corollary 1 . Indeed, suppose by way of contradiction that $0 \in W$, and let $f>0$ be the corresponding support function. Formula (1.10) is valid, hence $\Delta f-2 K f=0$. Now apply Koebe's theorem and Corollary 3 of [2]; we conclude that the universal covering surface of $M^{2}$ is parabolic, therefore $A=0$ and $\varphi\left(M^{2}\right)$ is the plane.

Immersion in the sphere. In this section we shall investigate what can be salvaged of Theorem 1 in the case of an immersion of $M^{2}$ into a sphere.

We begin by considering locally an isometric immersion $\varphi: M^{n} \rightarrow S^{k+n}, k \geqslant 1$. The sphere $S^{k+n}$ is viewed as the unit hypersphere of $R^{k+n+1}$ centered at the origin; by $x$ we denote its position vector, which is also its outward unit normal. 
Let $a$ be a fixed unit vector in $R^{k+n+1}$. For $p \in M^{n}$ we consider the vector $x-a$ with initial point $a \in S^{k+n}$ and endpoint $\varphi(p)$; we decompose it uniquely as $x-a=x_{\nu}+x_{T}+x_{N}$, where $x_{\nu}$ is its component normal to $S^{k+n}, x_{T}$ the component tangent to $\varphi\left(M^{n}\right)$, and $x_{N}$ the component normal to $\varphi\left(M^{n}\right)$ and tangent to $S^{k+n}$. We rewrite this equation as

$$
x_{T}=-a+\langle a, x\rangle x-x_{N}
$$

We now define

$$
W=S^{k+n}-\bigcup_{p} S_{p}^{n}
$$

where $S_{p}^{n}$ is the great $n$-sphere in $S^{k+n}$ which is tangent to $\varphi\left(M^{n}\right)$ at $\varphi(p)$. Suppose $x_{N}=0$ at $\varphi(p)$; then, either $x-a=0$ there, so $a \notin W$, or $x-a \neq 0$, in which case $x-a$ is not perpendicular to $S^{k+n}$ at its endpoint; hence $x_{T}+x_{N} \neq 0$, so $x_{T} \neq 0$. It now follows from (2.1) that the geodesic through $x_{T}$ lies in the plane determined by $a$ and $x$; in other words, there is a great circle tangent to $\varphi\left(M^{n}\right)$ which passes through $a$; so again $a \notin W$. We have shown that $a \in W$ implies $x_{N} \neq 0$ on $M^{n}$. It is geometrically obvious that the converse also holds.

We assume from now on that $a \in W$. If we set $x_{N}=f e, f>0,|e|=1$, then (2.1) implies $f=-\langle a, e\rangle$. By analogy to the euclidean case, we call $e$ the canonical normal vectorfield, and $f$ the canonical support function of $M^{n}$ corresponding to the point $a$.

The expression

$$
x_{T}=-a+\langle a, x\rangle x+\langle a, e\rangle e
$$

for the vectorfield $x_{T}$ tangent to $\varphi\left(M^{n}\right)$ will be the starting point for the subsequent computations; we shall only sketch them because they are similar to those in the euclidean case.

RemarK 4. Suppose $\varphi: M^{n} \rightarrow S^{n+1}$ is an immersion and $e$ is unit normal vectorfield of $M^{n}$ in $S^{n+1}$. We do not assume $W \neq \varnothing$, but define $x_{T}$ by (2.2); then $\left\langle x_{T}, x\right\rangle=0$ and $\left\langle x_{T}, e\right\rangle=0$, so $x_{T}$ is a tangent vectorfield of $M^{n}$. It follows that all the subsequent numbered equations, which we derive on the basis of (2.2), will be valid also for an oriented hypersurface of a sphere.

We differentiate covariantly equation (2.2) in the direction of an arbitrary tangent vector $X$ of $M^{n}$. Using the Gauss formula for $S^{k+n}$ in $R^{k+n+1}$ and for $M^{n}$ in $S^{k+n}$, as well as the Weingarten formula for the direction $e$ in $S^{k+n}$, we obtain an equation whose tangential component is

$$
\nabla_{X} x_{T}=(\langle a, x\rangle I+f A) X
$$

here $\nabla$ is the connection on $M^{n}$, and $A$ the Weingarten mapping for the direction $e$ on $S^{k+n}$. Therefore,

$$
\operatorname{div} x_{T}=n\langle a, x\rangle+f \operatorname{trace} A .
$$

The normal component gives the same equation as in the euclidean case:

$$
\alpha\left(X, x_{T}\right)+\langle X, \operatorname{grad} f\rangle e+f \nabla_{X}^{\perp} e=0 ;
$$


here $\alpha$ denotes the second fundamental form of $M^{n}$ in $S^{k+n}$, and $\nabla^{\perp}$ the connection in the normal bundle in $S^{k+n}$. Multiplication of (2.4) by $e$ yields

$$
\operatorname{grad} f=-A x_{T} \text {. }
$$

We take a local orthonormal frame field $\left\{e_{1}, \ldots, e_{k}\right\}$ of the normal bundle of $M^{n}$, with $e_{1}=e$, and denote by $A_{\beta}$ the Weingarten mapping for the direction $e_{\beta}$. Then (1.7) holds in our context as well, because (1.5) and (1.6) do.

From now on we additionally assume $e$ is parallel in the normal bundle in $S^{k+n}$. Now (1.7) reduces to

$$
A_{\beta} x_{T}=0, \quad \beta \geqslant 2 \text {. }
$$

We compute the Laplacian of $f$ and, using $\nabla_{X}^{\perp} e=0$, find

$$
\Delta f+\operatorname{trace}\left(A^{2}\right) f+\operatorname{trace} A\langle a, x\rangle=\langle a, \operatorname{grad}(\operatorname{trace} A)\rangle .
$$

This equation is stated in [7, p. 489] for the case $\varphi: M^{n} \rightarrow S^{n+1}$.

We mention here in passing that (2.7) contains a proof of the following result due to A. V. Pogorelov [8, Theorem 1]: Let $M^{n}$ be a compact minimal hypersurface in $S^{n+1}$ with $W \neq \varnothing$; then $M^{n}$ is a great hypersphere. Indeed, (2.7) reduces to $\Delta f+\operatorname{trace}\left(A^{2}\right) f=0$ in this case and, taking a point in $W$ as origin, we have $f>0$; now $f=$ const by the maximum principle, so $A=0$.

For immersions $\varphi: M^{2} \rightarrow S^{k+2}$, the best we can expect is that a theorem analogous to Theorem 1 holds; that is, if $a \in W$ and the corresponding $e$ is a minimal section, then $\varphi\left(M^{2}\right)$ is totally geodesic. However, this is false in arbitrary codimension: take a complete minimal surface $M^{2}$ in $S^{3}$, and consider $S^{3}$ as a great sphere in $S^{4}$; if $a$ denotes the north pole in $S^{4}$, then $a \in W$ and the corresponding normal field is the constant vector $-a$; therefore, $e$ is parallel and $A=0$. Still, this example hints at what we can reasonably hope for: a reduction of codimension of the immersion.

THEOREM 2. Let $M^{2}$ be a complete two-dimensional manifold and $\varphi: M^{2} \rightarrow S^{k+2}$ an isometric immersion. Suppose there is a point in $W$ with respect to which the canonical normal vectorfield is a minimal section; then $\varphi$ immerses $M^{2}$ into a great hypersphere of $S^{k+2}$.

Proof. It is sufficient to prove our assertion for the universal Riemannian covering surface of $M^{2}$; so we suppose $M^{2}$ is simply connected.

If $a \in W$ is the point in question, $e$ the corresponding canonical normal vectorfield and $A$ the corresponding Weingarten mapping, equations (2.2)-(2.6) are valid and $(2.7)$ reduces to

$$
\Delta f+\operatorname{trace}\left(A^{2}\right) f=0, \quad f=-\langle a, e\rangle>0 .
$$

We again consider the subset $C$ of $M^{2}$ where $x_{T}$ vanishes. On $M^{2}-C$ we have locally det $A_{\beta}=0, \beta \geqslant 2$. Therefore, the Gauss equation takes the form $K=1+$ $\operatorname{det} A$ on $M^{2}-C$ and, using trace $A=0$, we rewrite this as

$$
\operatorname{trace}\left(A^{2}\right)=-2 K+2 \text {. }
$$


First assume $C$ has empty interior. Then (2.9) holds by continuity on all of $M^{2}$, and (2.8) reads

$$
\Delta f-2 K f+2 f=0 .
$$

By the uniformization theorem for Riemann surfaces, we have the following alternatives: either $M^{2}$ is compact or parabolic; then $f=$ const, so $\operatorname{trace}\left(A^{2}\right)=0$ and hence $A=0$; or $M^{2}$ is hyperbolic; however, this is impossible according to Corollary 3 of [2].

Since $A=0$ and $e$ is parallel in the normal bundle, $e$ is a constant vector in $R^{k+3}$. It follows that the function $g: M^{2} \rightarrow R, g(p)=\langle x(p), e\rangle$, has derivative zero in every direction $X \in T M^{2}$, so $g=$ const. This means geometrically that $\varphi\left(M^{2}\right)$ lies in a $(k+2)$-dimensional linear variety $L$ of $R^{k+3}$, which is perpendicular to $e$. So $\varphi\left(M^{2}\right)$ lies in the hypersphere $S^{k+1}=L \cap S^{k+2}$ of $S^{k+2}$. Because the constant vector $e$ is tangent to $S^{k+2}$, this sphere $S^{k+1}$ must be a great hypersphere of $S^{k+2}$.

Now assume $C$ has interior points; then we conclude from (2.5) that $f=$ const $>0$ on an open set. Therefore $A=0$ on an open set by $(2.8)$. Let $(u, v)$ be local isothermal parameters on $M^{2}, U=\partial / \partial u$ and $V=\partial / \partial v$ the corresponding coordinate vector fields. If we set $z=u+i v$ and

$$
F(z)=\frac{1}{2}(\langle A U, U\rangle-\langle A V, V\rangle)-i\langle A U, V\rangle,
$$

then $F(z) d z^{2}$ is a global holomorphic quadratic differential on $M^{2}$; this is a well-known consequence of the hypothesis that $e$ is a minimal section. Clearly, $F(z) d z^{2}$ vanishes exactly at the points where $A=0$, so we have $A=0$ on all $M^{2}$. As we just showed, this means $\varphi\left(M^{2}\right)$ lies in a great hypersphere of $S^{k+2}$. The proof is complete.

In the case of a minimal surface in the 3-sphere, we have the analogue of Corollary 1.

CoROllary 2. Let $M^{2}$ be a complete surface and $\varphi: M^{2} \rightarrow S^{3}$ an isometric minimal immersion with the property that the union of all the great $S^{2}$ tangent to $\varphi\left(M^{2}\right)$ does not cover all $S^{3}$; then $\varphi$ is an imbedding of $M^{2}$ onto a great $S^{2}$.

Proof. We may assume $M^{2}$ is orientable. If $a \in W$ then the corresponding normal vectorfield $e$ is automatically parallel in the normal bundle. Thus, $\varphi$ maps $M^{2}$ into a great $S^{2}$ according to Theorem 2. Since $A=0$ we have $K=1$, therefore $M^{2}$ is compact and simply connected. Now we argue in the usual way. Because $\varphi\left(M^{2}\right)$ is both open and closed, $\varphi$ maps $M^{2}$ onto $S^{2}$. Because $\varphi: M^{2} \rightarrow S^{2}$ is a local diffeomorphism and $M^{2}$ is compact, $\varphi$ is a covering projection, hence it is a diffeomorphism.

We observe that, in the case of an immersion $\varphi: M^{2} \rightarrow S^{3}$, the normal field $e$ describes the spherical image of $\varphi\left(M^{2}\right)$, that is, the image of $M^{2}$ under the Gauss mapping $p \rightarrow e(p) \in S^{3}$; here the vector $e(p)$ is translated in the ambient euclidean space so as to have initial point at the origin. From this point of view, the condition $f>0$ means the spherical image of $\varphi\left(M^{2}\right)$ lies in an open hemisphere. This led us to the following reformulation and sharpening of Corollary 2. 
THEOREM 3. Let $M^{2}$ be a complete oriented surface and $\varphi: M^{2} \rightarrow S^{3}$ an isometric minimal immersion. If the spherical image of $\varphi\left(M^{2}\right)$ lies in a closed hemisphere, then $\varphi$ is an imbedding of $M^{2}$ onto a great $S^{2}$.

Proof. The hypothesis on the spherical image means there exists some unit vector $a$ in $R^{4}$ with the property $f(p)=-\langle a, e(p)\rangle \geqslant 0$ for all $p \in M^{2}$; here $e$ denotes a global unit normal vectorfield of $M^{2}$ in $S^{3}$. First assume the spherical image of $\varphi\left(M^{2}\right)$ lies in an open hemisphere. So $f>0$, which implies $a \in W$. Since $W$ is not empty, Corollary 2 applies and the assertion follows.

If the spherical image lies in a closed hemisphere and has a point on the boundary, then $f \geqslant 0$ and $f\left(p_{0}\right)=0$ for some $p_{0}$. On the other hand, (2.8) is valid so $\Delta f \leqslant 0$. By E. Hopf's maximum principle, $f$ is identically zero. At this point we can finish the proof by appealing to Theorem 1 of Nomizu and Smyth [7], or we may proceed as follows. According to Remark 4, (2.3) and (2.5) are still valid. Suppose $C$ has nonempty interior; then we deduce from (2.3) that $\langle a, x\rangle=0$ on an open set $U \subset M^{2}$. Thus, $\varphi(U)$ lies in a great $S^{2}$ so $A=0$ on $U$. By analyticity, as in the proof of Theorem 2, we have $A=0$ on $M^{2}$; therefore, $\varphi$ immerses $M^{2}$ into a great $S^{2}$ and we complete the proof just as in Corollary 2. If $C$ has empty interior, we use (2.5) to obtain $\operatorname{det} A=0$ on a dense set. Since trace $A=0$, we again have $A=0$ on $M^{2}$ and we proceed as before.

De Giorgi [3], and later Simons [9], proved that a compact minimal hypersurface of the sphere must be a great hypersphere if its spherical image lies in an open hemisphere. Nomizu and Smyth [7] showed that the conclusion still holds if the spherical image lies in a closed hemisphere. Theorem 3 is an improvement of this last result in dimension two.

\section{REFERENCES}

1. B.-Y. Chen, Geometry of submanifolds, Dekker, New York, 1973.

2. D. Fischer-Colbrie and R. Schoen, The structure of complete stabie minimal surfaces in 3-manifolds of non-negative scalar curvature, Comm. Pure Appl. Math. 33 (1980), 199-211.

3. E. de Giorgi, Una estensione del teorema di Bernstein, Ann. Scuola Norm. Sup. Pisa (3) 19 (1965), $79-85$.

4. B. Halpern, On the immersion of an $n$-dimensional manifold in $(n+1)$-dimensional Euclidean space, Proc. Amer. Math. Soc. 30 (1971), 181-184.

5. D. A. Hoffman, R. Osserman and R. Schoen, On the Gauss map of complete surfaces of constant mean curvature in $R^{3}$ and $R^{4}$ (typescript).

6. J. A. Little, On singularities of submanifolds of higher dimensional Euclidean spaces, Ann. Mat. Pura Appl. (4) 83 (1964), 261-335.

7. K. Nomizu and B. Smyth, On the Gauss mapping for hypersurfaces of constant mean curvature in the sphere, Comm. Math. Helv. 44 (1969), 484-490.

8. A. V. Pogorelov, On minimal hypersurfaces in spherical space, Soviet Math. Dokl. 13 (1972), $1218-1219$.

9. J. Simons, Minimal varieties in Riemannian manifolds, Ann. of Math. (2) 88 (1968), 62-105.

10. B. Smyth, Submanifolds of constant mean curvature, Math. Ann. 205 (1973), 265-280.

Department of Mathematics, University of Ioannina, Ioannina, Greece 